\title{
Time to colonoscopy, cancer probability, and precursor lesions in the Danish colorectal cancer screening program
}

This article was published in the following Dove Press journal: Clinical Epidemiology

\author{
Lasse Kaalby iD ${ }^{1,2}$ \\ Morten Rasmussen (iD ${ }^{3}$ \\ Erik Zimmermann-Nielsen (iD) ' \\ Magdalena Maria Buijs (iD) \\ Gunnar Baatrup (D) ${ }^{1,2}$ \\ 'Department of Surgery, Odense \\ University Hospital, Odense, Denmark; \\ ${ }^{2}$ Department of Clinical Science, \\ University of Southern Denmark, \\ Odense, Denmark; ${ }^{3}$ Department of \\ Digestive Diseases K, Bispebjerg \\ Hospital, Copenhagen, Denmark
}

Correspondence: Lasse Kaalby

House of Research, Svendborg Hospital, Baagøes Alle 15, 5700 Svendborg,

Denmark

Tel +45 28777610

Email LKM@rsyd.dk
Purpose: The aim of this study was to investigate the effect of response time from the Fecal Immunochemical Test (FIT) based screening invitation to the conclusive screening Optical Colonoscopy (OC) on the risk of detecting colorectal cancer (CRC), advanced stage disease and precursor lesions.

Patients and methods: We used a cross-sectional study design and included all 62,554 screening participants registered in the Danish Colorectal Cancer Screening Database who tested FIT-positive between March 2014 and December 2016. The main exposure was response time, measured as the time from initial invitation to the conclusive OC. Our main outcomes were the probability of being diagnosed with CRC, advanced stage disease or precursor lesions.

Results: Of the 62,554 FIT-positive participants, 53,171 (85\%) received an OC and were eligible for analysis (median age 63.7 years, $56 \%$ men). In this group, 3,639 cancers were registered, 2,890 of which were registered with a defined stage of disease (79\%), and 1,042 (36\%) of these were advanced stage (UICC III \& IV). In addition, 17,732 high-risk and 10,605 low-risk adenomas were identified. Compared to participants receiving the conclusive examination within 30 days, those receiving the examination more than 90 days after initial invitation were 3.49 times more likely to be diagnosed with any CRC (OR 3.49 [95\% CI, 3.13-3.89]) and 2.10 times more likely to have advanced stage disease (OR 2.10 [95\% CI, 1.73-2.56]). Those waiting for the longest were also more likely to have one or more high-risk adenomas (OR 1.59 [95\% CI, 1.50-1.68]).

Conclusion: Increased screening response time was associated with a higher probability of detecting high-risk adenomas, any stage CRC and advanced stage cancer. More research is needed to explain what causes these associations.

Keywords: colorectal cancer screening, screening response time, delayed participation, screening uptake

\section{Introduction}

Colorectal cancer (CRC) is a common malignancy in both Denmark and the Western world. ${ }^{1}$ Screening has shown to reduce the CRC-specific mortality. ${ }^{2-5}$ The use of fecal occult blood test (FOBT) is recommended as the primary tool of screening by the European Guidelines for Quality Assurance in CRC screening and diagnosis. ${ }^{6}$ In case of a positive FOBT, participants subsequently receive a colonic examination which is generally an Optical Colonoscopy (OC).

The Danish screening program was initiated in March 2014. Participation is free of charge and citizens aging from 50 to 74 years are invited on equal terms. The 
screening program uses (Fecal Immunochemical Test [FIT]; OC Sensor System; Eiken Chemical Company, Tokyo, Japan) to detect traces of blood in stool samples as the primary tool of inclusion. The cut-off value in Denmark is established at $100 \mathrm{ng}$ hemoglobin $/ \mathrm{mL}$ buffer (equal to $20 \mu \mathrm{g} \mathrm{hgb./g} \mathrm{feces).}{ }^{7}$ In the Dutch screening program, the cut-off value was increased from 15 to 47 $\mu \mathrm{g} \mathrm{hgb./g} \mathrm{feces} \mathrm{due} \mathrm{to} \mathrm{higher} \mathrm{participation} \mathrm{rate} \mathrm{and} \mathrm{lower}$ positive predictive value (PPV) than predicted. ${ }^{8}$ Brenner et $\mathrm{al}$, argue that a cut-off value of $15 \mu \mathrm{g} \mathrm{hgb./g}$ feces would ensure a specificity of $95 \%$ and a PPV of $47 \%$ for the detection of advanced neoplasia during subsequent diagnostic OC. ${ }^{9}$

The FIT is characterized by a higher sensitivity compared to earlier tests such as the Guaiac-based FOBT, low costs, and simple distribution options. ${ }^{10}$ Imperiale et al, reported a sensitivity for detecting $\mathrm{CRC}$ at $73.8 \%$ and $23.8 \%$ for advanced precursor lesions with a specificity at nearly $95 \%$ in 9,989 persons investigated by OC, who had submitted a FIT test with a cut-off value equal to the one used in the Danish CRC screening program. ${ }^{11}$

In Denmark, OC is offered to all FIT-positive participants. FIT and OC uptake, both in screening and in general, is known to be affected by a number of factors, including age, gender, and social deprivation. ${ }^{12-16}$ Both FIT and OC performance may have consequences for the success of the screening program. A delay in uptake might hold potential for neoplastic progression, as suggested by Corley et al. ${ }^{17} \mathrm{~A}$ few other studies addressed this problem, however small samples limited the power. ${ }^{18,19}$ Furthermore, all these studies examined the time from positive FOBT result to final OC, and do not include the time from initial screening invitation to final OC. The causes and consequences of prolonged time from the initial screening invite to a positive FOBT have, to our knowledge not yet been investigated. The objective of this study was to evaluate whether extended response time to the conclusive screening examination impacts the probability of being diagnosed with $\mathrm{CRC}$ or precursor lesions.

\section{Materials and methods}

We conducted a cross-sectional study using a registerbased approach. All individual-level data were linked using the Danish civil registration number, a unique identifier given at birth or immigration to all residents by the Civil Registration System. The study population consisted of all FIT-positive screening participants in Denmark between March 2014 and December 2016. Invitations were sent to all citizens between 50 and 74 years of age, resulting in a total of $1,437,836$ invitations in this period. $899,411(62.6 \%)$ accepted the invitation by submitting a fecal sample, of which 62,554 tested FIT-positive (6.9\%). ${ }^{7}$

All screening invitees receive a FIT-kit containing sample tubes and stool collection tools, along with a written invitation in the mail, followed by two reminders. A positive FIT is followed by a written invitation to OC within two weeks sent both electronically to a secure personal inbox and by mail. The invitation contains an appointment for OC as well as patient information regarding the procedure. The results are discussed with the participant after OC. Participants with an incomplete OC are referred to CT colonography. Detection of polyps, cancer, or stenosis will result in a second OC in propofol sedation or general anesthesia. All pathology results will be sent by letter, in case of unexpected findings the participant will be invited to the out-patient clinic. Depending on the findings, participants are contacted either electronically or by phone after pathology results are obtained.

Known factors of incomplete OC investigations include poor bowel cleansing, inflammatory bowel disease, female gender, and history of abdominal or pelvic surgery. ${ }^{20,21}$

A positive CT colonography will be followed up by a second OC in propofol sedation or general anesthesia.

From 2014 till 2017 the national screening program was rolled out, and now invites all citizens in the designated age-group biannually.

\section{Cancer outcomes and time to conclusive examination}

Four different outcomes were analyzed separately: detection of any CRC, advanced stage CRC, high-risk adenomas, and low-risk adenomas. Screen-detected CRC was defined as pathologically confirmed cancer in the colon or rectum detected during a screening examination. The CRC stage was registered according to the TNM classification (UICC 8th edition). Advanced stage CRC was defined as UICC stage III and IV. High-risk adenomas were defined as one adenoma $\geq 10 \mathrm{~mm}$ in diameter, three or more adenomas, tubulovillous/villous histology, and/or high-grade dysplasia. Low-risk adenomas were defined as fewer than three adenomas, $<10 \mathrm{~mm}$ in diameter and tubular histology with lowgrade dysplasia. All participants were registered once, by their most "severe" pathology.

The exposure in this study was defined as time elapsing from invitation to conclusive OC. Elapsed time was treated as a continuous variable divided into four intervals: 
The reference was $\leq 30$ days and categories of comparison were $31-60$ days, $61-90$ days, and $>90$ days, respectively. These intervals were chosen to balance sample sizes on outcome distributions and to meet monthly intervals as practical cut-offs. The year of invitation was added as a variable containing the categories 2014, 2015, and 2016.

The results were adjusted for age $(50-54,55-59,60$ $64,65-69$, and 70-75), gender and year of invitation were included in the analysis.

\section{Data sources}

All data were obtained from the Danish Colorectal Cancer Screening Database (DCCSD), a clinical quality assessment database established to monitor the program. The database merges data from other registers: The Danish National Patient Registry, The National Pathology Registry, The Danish Colorectal Cancer Group, and The Invitation and Administration Module for the screening program. All data are gathered electronically, and no manual entering of data is performed directly into the DCCSD. No data concerning cancer location, ie, rectal or colonic, or synchronisms were available. Data from 2017 were not obtainable at the time of data extraction (05-02-2018).

\section{Statistical methods}

A multivariable logistic regression was used to assess the outcome probability and to adjust for confounding effects.
Adjusted odds ratios (ORs) and 95\% confidence intervals were used to present estimates. Observations with missing data were excluded from the main analysis, but were not excluded from the study.

The latest available date of the conclusive examination was chosen to ensure that the registered procedure was completed. The sensitivity analysis included changing the groups of exposure to different response times, so that exposure was either the time from invitation to positive-FIT result (the date the lab received the sample) or the time from a positive FIT (lab-date) to the latest performed OC.

Analyses were performed in STATA version 15.0. (StataCorp LLC, College Station, Texas, USA).

\section{Ethics}

Due to the register-based approach, no approval was required from the National Committee on Health Research Ethics. The study has been reported to the Danish Data Protection Agency, journal 18/2913. All data were stored in accordance with Danish legislation and were pseudoanonymized before analysis.

\section{Results}

From 2014 to 2016, 62,554 screening participants tested FIT-positive (6.9\%), of these 57,370 (91.7\%) participants accepted the invitation for OC (Table 1). A total of 4,100 $(6.6 \%)$ participants were registered without a conclusive

Table I Characteristics among screening participants testing FIT-positive followed by OC $(\mathrm{N}=57,370)$

\begin{tabular}{|c|c|c|c|c|c|}
\hline \multirow{2}{*}{$\begin{array}{l}\text { Characteristics } \\
\text { Days }\end{array}$} & \multicolumn{5}{|c|}{ Time-to-examination (\%) } \\
\hline & $\leq \mathbf{3 0}$ & $31-60$ & $61-90$ & $>90$ & Total \\
\hline Total participants & $11,060(19.3 \%)$ & $24,244(42.3 \%)$ & $10,237(17.8 \%)$ & II,829 (20.6\%) & $57,370(100 \%)$ \\
\hline \multicolumn{6}{|l|}{ Gender } \\
\hline Women & $4,991(45.1)$ & I I,064 (45.6) & $4,404(43.0)$ & $4,798(40.6)$ & $25,257(44.0)$ \\
\hline Men & $6,069(54.9)$ & $13,180(54.4)$ & $5,833(57.0)$ & $7,031(59.4)$ & $32,113(56.0)$ \\
\hline \multicolumn{6}{|l|}{ Age, years } \\
\hline $50-54$ & $1,304(I 1.8)$ & $4,056(16.7)$ & $2,440(23.8)$ & $2,533(21.4)$ & $10,333(18.0)$ \\
\hline $55-59$ & $1,340(12.1)$ & $3,170(13.1)$ & $1,530(15.0)$ & I,583 (13.4) & $7,623(13.3)$ \\
\hline $60-64$ & $\mathrm{I}, 854(16.8)$ & $4,203(17.3)$ & $1,713(16.7)$ & $1,859(15.7)$ & $9,629(16.8)$ \\
\hline $65-69$ & $2,639(23.9)$ & $5,208(21.5)$ & $1,946(19.0)$ & $2,42 I(20.5)$ & $|2,2| 4(2 \mid .3)$ \\
\hline$\geq 70$ & $3,923(35.5)$ & $7,607(31.4)$ & $2,608(25.5)$ & $3,433(29.0)$ & $|7,57|(30.6)$ \\
\hline Median (IQR) & $63.7(49-76)$ & $63.7(49-76)$ & $63.7(49-76)$ & $63.7(49-76)$ & $63.7(49-76)$ \\
\hline \multicolumn{6}{|l|}{ Year of FIT-test } \\
\hline 2014 & $2,346(21.2)$ & $6,859(28.3)$ & $2,761(27.0)$ & $3,632(30.7)$ & I5,598 (27.2) \\
\hline 2015 & $3,905(35.3)$ & $8,466(34.9)$ & $3,563(34.8)$ & $4,077(34.5)$ & $20,011(34.9)$ \\
\hline 2016 & $4,809(43.5)$ & $8,919(36.8)$ & $3,913(38.2)$ & $4,120(34.8)$ & 21,761 (37.9) \\
\hline
\end{tabular}

Abbreviations: IQR, interquartile range; FIT, Fecal Immunochemical Test; OC, Optical Colonoscopy. 
outcome for OC and $99(0.2 \%)$ participants were registered with inconclusive examinations (Figure 1). This resulted in a population of $53,171(85.0 \%)$ participants eligible for analysis in this study.

A conclusive investigation was performed within 30 days in 11,060 (19.3\%) participants, within 31-60 days in $24,244(42.3 \%)$ participants, within $61-90$ days in 10,237 (17.8\%) participants, and after 90 days in 11,829 (20.6\%).

The median age was 63.7 years (Interquartile Range [IQR], 49-76 years), 25,257 (44.0\%) of participants were female and $79.4 \%$ underwent a conclusive investigation within 90 days of their initial invitation. The mean time from invitation to final OC in the group $>90$ days was 174 days (IQR, 91-1,348 days) and for the entire population 72.2 days (IQR, 10-1,348 days).

An increasing number of participants were over time receiving $\mathrm{OC}$ as the screening program was rolled out: $15,598(27.2 \%)$ in $2014,20,011(34.9 \%)$ in 2015 , and $21,761(37.9 \%)$ in 2016.

CRC was detected in 3,639 participants (Table 2). In addition, 17,732 participants with high-risk adenomas and 10,605 participants with low-risk adenomas were identified during screening. Of the 5,184 participants who did not receive further investigations, age and gender distributions were similar to the remaining population.

Compared to participants receiving the conclusive screening examination within 30 days after the initial invite, those receiving the examination after 31-60 days were 1.24 times more likely to be diagnosed with CRC (OR 1.24 [95\% CI, 1.11-1.39]) (Table 2). Those receiving the conclusive screening examination 31-60 days after invitation were also 1.57 times more likely to have a stage I CRC compared to the reference group (OR 1.57 [95\% CI, 1.271.96]) (Table S1). The probability of detecting advanced stage disease, high-risk adenomas, or low-risk adenomas were not associated with recieving a conclusive screening examination 31-60 days after initial invitation.

The group receiving a conclusive investigation 61-90 days after invitation had a significant higher probability of having CRC (OR 1.59 [95\% CI, 1.40-1.80]), advanced stage disease (OR 1.37 [95\% CI, 1.10-1.71]), and highrisk adenomas (OR 1.16 [95\% CI, 1.09-1.23]) compared to the reference group ( $<30$ days). The 61-90 days group had a lower probability for low-risk adenomas (OR 0.91 [95\% CI, 0.85-0.97)]. In the same group, the probability of stage I, II, and III CRC was higher than in the reference group (OR 2.16 [95\% CI, 1.70-2.74], OR 1.32 [95\% CI, 1.031.68], and OR 1.46 [95\% CI, 1.13-1.88], respectively).
In the group receiving the conclusive screening examination after 90 days, the same trend is seen as in the 61-90 days group. Participants were more likely to be diagnosed with CRC (OR 3.49 [95\% CI, 3.13-3.89]), advanced stage CRC (OR 2.10 [95\% CI, 1.73-2.56]), and high-risk adenomas (OR 1.59 [95\% CI, 1.50-1.68]). As in the 61-90 days, group participants were less likely to be diagnosed with a low-risk adenoma (OR 0.55 [95\% CI, 0.51-0.59]).

In the $>90$ days, group participants were more likely to be diagnosed with any-stage of $\mathrm{CRC}$, varying from a 3.58 OR for stage I (OR 3.58 [95\% CI, 2.89-4.45]) to a 1.66 OR in stage IV (OR 1.66 [95\% CI, 1.15-2.40]).

Accounting for gender, year of screening, and age moderately reduced the strength of the association, but did not change the direction of the results.

In the sensitivity analysis, the associations were tested for the elapsed time from both initial screening invitation and positive FIT to conclusive OC. The elapsed time from positive FIT to conclusive OC was found to influence the odds of being diagnosed with CRC by up to 5.32 (OR 5.32 [95\% CI, 4.89-5.79]) times among those receiving the investigation more than 90 days after positive FIT (Table S2). The time from initial invitation to positive FIT did not influence the probability of being diagnosed with CRC.

\section{Discussion}

In this FIT-positive screening population, the time from initial invitation to conclusive $\mathrm{OC}$ correlated positively to the probability of detecting $\mathrm{CRC}$, advanced stage disease and high-risk adenomas. For low-risk adenomas, a reverse trend was found. The time from initial invite to positive FIT did not appear to affect the probability of CRC in the sensitivity analysis, and cannot be ascribed a role to the increase in odds. In this study, the mean time from invitation to conclusive examination was 72.2 days, of which the mean time from invitation to positive sample was 38.9 days and the mean time from positive sample to OC was 33.3 days. Nearly $80 \%$ of all screening participants underwent the final examination within 90 days. The European guidelines for $\mathrm{CRC}$ screening recommend $\mathrm{OC}$ being performed within 31 days after a positive FIT. $^{6}$ Our findings suggest that high-risk individuals appear to have a delay to the conclusive screening examination, which might lead to neoplastic progression of the disease and thus influence the possibility of curative treatment.

The reason why there is an increase in neoplastic findings with increasing time to conclusive examination in this study is unclear. A possible explanation is that the different 


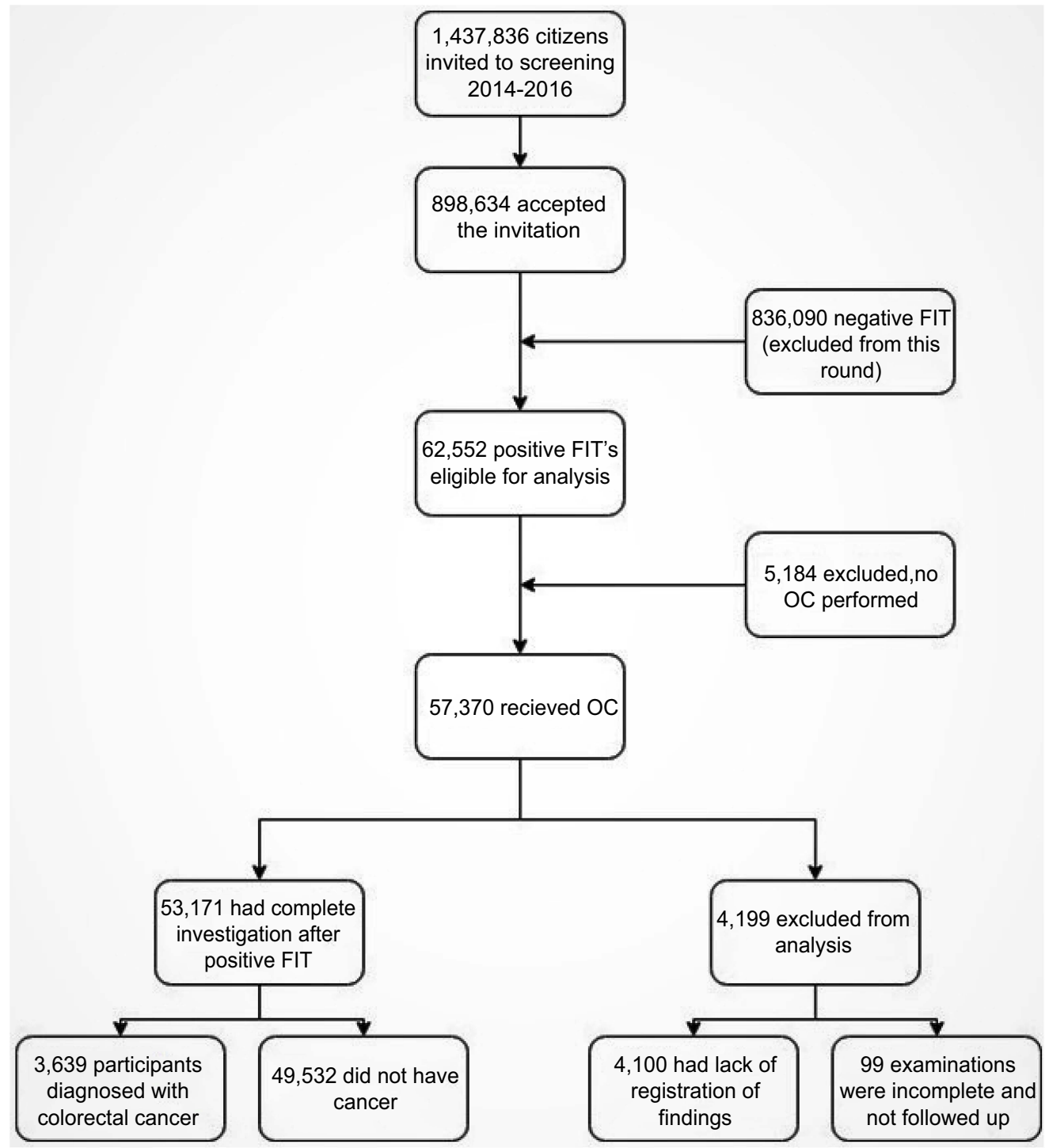

Figure I Flow of screening participants.

Abbreviations: FIT, Fecal Immunochemical Test; OC, Optical Colonoscopy.

groups of participants are likely to have a different demographic composition, rather than different neoplastic growth. A lower socioeconomic status, gender, and increased age have been found to negatively affect CRC screening uptake ${ }^{12-14}$ and might explain the delay presented in our population as well.

Personal factors of the participants might also cause a delay in uptake, such as psychological impact (perceived susceptibility and severity), structural issues (eg, distance to hospital, OC capacity), or attitude toward screening. ${ }^{22,23}$

Corley et al, performed a similar study focusing mainly on time from positive FIT to conclusive OC. ${ }^{17}$ They concluded that an OC after more than 10 months was associated with a higher risk of CRC and advanced stage disease. Our findings are consistent with these findings and support their argument that elapsed time to screening should be considered as a variable in future studies.

Strengths of this study are a large and diverse study population extracted from the Danish national databases, a large number of pathologically validated neoplasia outcomes, and absence of selection-bias since all citizens in the designated age-group were invited.

A limitation of this study is the validity of the data from the DCCSD, especially due to errors in registration and the pooling-process from other databases. It is of concern that 4,100 screening participants are not registered in the database with a conclusive finding. This may be caused by errors in the 
Table 2 Odds ratios of OC-outcomes as time to conclusive examination increases in screening participants

\begin{tabular}{|c|c|c|c|}
\hline Time to examination & No. of cases (\%) & Adjusted odds ratio $(95 \% \mathrm{CI})^{\mathrm{a}}$ & Unadjusted odds ratio $(95 \% \mathrm{Cl})$ \\
\hline \multicolumn{4}{|c|}{ Colorectal cancer $(n=3,639)$} \\
\hline$\leq 30$ days & $458(4.1)$ & I (reference) & I (reference) \\
\hline $3 \mathrm{I}-60$ days & $\mathrm{I}, \mathrm{I} 78(4.9)$ & $1.24(1.11-1.39)$ & $1.18(1.06-1.32)$ \\
\hline $6 I-90$ days & $578(5.7)$ & $1.59(1.40-1.80)$ & $1.39(1.22-1.57)$ \\
\hline$>90$ days & $\mathrm{I}, 425(\mathrm{I} 2.1)$ & $3.49(3.13-3.89)$ & $3.17(2.84-3.53)$ \\
\hline \multicolumn{4}{|c|}{$\begin{array}{l}\text { Advanced stage colorectal cancer } \\
(n=I, 042)\end{array}$} \\
\hline$\leq 30$ days & $158(1.4)$ & I (reference) & I (reference) \\
\hline $3 \mathrm{I}-60$ days & $380(1.6)$ & $1.14(0.95-1.38)$ & $1.10(0.91-1.32)$ \\
\hline $6 I-90$ days & $178(1.7)$ & $1.37(1.10-1.71)$ & $1.22(0.98-\mid .5 I)$ \\
\hline$>90$ days & $326(2.8)$ & $2.10(1.73-2.56)$ & $1.96(1.61-2.37)$ \\
\hline \multicolumn{4}{|c|}{ High-risk adenomas $(n=\mid 7,732)$} \\
\hline$\leq 30$ days & $3,161(28.6)$ & I (reference) & I (reference) \\
\hline $31-60$ days & $6,985(28.8)$ & $1.01(0.99-1.10)$ & $1.01(0.96-1.06)$ \\
\hline $6 I-90$ days & $3,105(30.3)$ & $1.16(1.09-1.23)$ & $1.09(1.03-1.15)$ \\
\hline$>90$ days & $4,472(37.8)$ & $1.59(1.50-1.68)$ & $\mathrm{I} .52(\mathrm{I} .44-1.6 \mathrm{I})$ \\
\hline \multicolumn{4}{|c|}{ Low-risk adenomas $(n=10,605)$} \\
\hline$\leq 30$ days & $2,268(20.5)$ & I (reference) & I (reference) \\
\hline $31-60$ days & $4,887(20.2)$ & $0.97(0.92-1.03)$ & $0.98(0.93-1.03)$ \\
\hline $6 I-90$ days & $1,960(19.1)$ & $0.91(0.85-0.97)$ & $0.92(0.86-0.98)$ \\
\hline$>90$ days & I,490 (I2.6) & $0.55(0.5 \mathrm{I}-0.59)$ & $0.56(0.52-0.60)$ \\
\hline
\end{tabular}

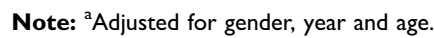

Abbreviation: OC, Optical Colonoscopy.

registration from the hospitals or in the merging process from the DCCSD with other databases.

Another limitation is that the UICC stage of $21 \%$ of the cancers was not registered in the DCCSD. A likely explanation is that this stage is dependent on pathologic evaluation, which can be complicated in case of piecemeal resections of large polyps, and complete response after chemo- or radiotherapy in rectal cancers. Therefore, it is possible that the group with missing stage registration differs from our study population, which should be considered in follow-up studies.

Our group is planning further studies examining the composition of the study population in more detail in order to detect which factors contribute to a delay of conclusive OC and should be addressed in CRC screening programs.

\section{Conclusion}

There is an association between elapsed time from initial screening invitation to conclusive examination and an increased overall probability of being diagnosed with CRC, advanced stage disease, and high-risk adenomas in the Danish screening program. Explanations for this association will be investigated in follow-up studies.

\section{Acknowledgments}

Odense Patient data Explorative Network (OPEN) has assisted in the making of this article by providing storage and support for the data management. This project has been funded by the Danish Cancer Society under Grant R100-A6747.

\section{Disclosure}

The authors report no conflicts of interest in this work.

\section{References}

1. Iversen LH, Green A, Ingeholm P, Osterlind K, Gogenur I. Improved survival of colorectal cancer in Denmark during 2001-2012 - the efforts of several national initiatives. Acta Oncol. 2016;55(Suppl 2):10-23. doi:10.3109/0284186X.2015.1131331

2. Fitzpatrick-Lewis D, Ali MU, Warren R, Kenny M, Sherifali D, Raina P. Screening for colorectal cancer: a systematic review and metaanalysis. Clin Colorectal Cancer. 2016;15(4):298-313. doi:10. 1016/j.clcc.2016.03.003

3. Kronborg O, Fenger C, Olsen J, Jorgensen OD, Sondergaard O. Randomised study of screening for colorectal cancer with faecaloccult-blood test. Lancet (London, England). 1996;348(9040):14671471. doi:10.1016/S0140-6736(96)03430-7

4. Shaukat A, Mongin SJ, Geisser MS, et al. Long-term mortality after screening for colorectal cancer. N Engl J Med. 2013;369(12):11061114. doi:10.1056/NEJMoa1300720 
5. Scholefield JH, Moss SM, Mangham CM, Whynes DK, Hardcastle JD. Nottingham trial of faecal occult blood testing for colorectal cancer: a 20year follow-up. Gut. 2012;61(7):1036-1040. doi:10.1136/gutjnl-2011300774

6. von Karsa L, Patnick J, Segnan N, et al. European guidelines for quality assurance in colorectal cancer screening and diagnosis: overview and introduction to the full supplement publication. Endoscopy. 2013;45(1):51. doi:10.1055/s-0032-1325997

7. Danish Colorectal Cancer Screening Database. Dansk Tarmkraftscreeningsdatabase Arsrapport 2016 [Annual Report of the Danish Colorectal Cancer Screening Database 2016]. København: Dansk tarmkræftscreeningsdatabase (DTS); December 2017.

8. Toes-Zoutendijk E, van Leerdam ME, Dekker E, et al. Real-time monitoring of results during first year of dutch colorectal cancer screening program and optimization by altering fecal immunochemical test cut-off levels. Gastroenterology. 2017;152(4):767-775.e762. doi:10.1053/j.gastro.2016.11.022

9. Brenner H, Werner S. Selecting a cut-off for colorectal cancer screening with a fecal immunochemical test. Clin Transl Gastroenterol. 2017;8(8):e111. doi:10.1038/ctg.2017.37

10. Stracci F, Zorzi M, Grazzini G. Colorectal cancer screening: tests, strategies, and perspectives. Front Public Health. 2014;2:210. doi:10.3389/fpubh.2014.00210

11. Imperiale TF, Ransohoff DF, Itzkowitz SH, et al. Multitarget stool DNA testing for colorectal-cancer screening. $N$ Engl J Med. 2014;370(14):1287-1297. doi:10.1056/NEJMoa1311194

12. Steele RJ, Kostourou I, McClements P, et al. Effect of gender, age and deprivation on key performance indicators in a FOBT-based colorectal screening programme. J Med Screen. 2010;17(2):68-74. doi: $10.1258 /$ jms.2010.009120

13. Morris S, Baio G, Kendall E, et al. Socioeconomic variation in uptake of colonoscopy following a positive faecal occult blood test result: a retrospective analysis of the NHS Bowel Cancer Screening Programme. Br J Cancer. 2012;107(5):765-771. doi:10.1038/bjc.2012.303

14. Ferrat E, Le Breton J, Veerabudun K, et al. Colorectal cancer screening: factors associated with colonoscopy after a positive faecal occult blood test. Br J Cancer. 2013;109(6):1437-1444. doi:10.1038/bjc.2013.476
15. Deding U, Henig AS, Salling A, Torp-Pedersen C, Boggild $\mathrm{H}$. Sociodemographic predictors of participation in colorectal cancer screening. Int J Colorectal Dis. 2017;32(8):1117-1124. doi:10.1007/ s00384-017-2832-6

16. Deding U, Henig AS, Hindersson P, Torp-Pedersen C, Bøggild H. Determinants of non-participation in colon examination following positive stool sample in colorectal cancer screening. Eur J Public Health. 2019. doi:10.1093/eurpub/ckz072

17. Corley DA, Jensen CD, Quinn VP, et al. Association between time to colonoscopy after a positive fecal test result and risk of colorectal cancer and cancer stage at diagnosis. JAMA. 2017;317(16):16311641. doi:10.1001/jama.2017.3634

18. Gellad ZF, Almirall D, Provenzale D, Fisher DA. Time from positive screening fecal occult blood test to colonoscopy and risk of neoplasia. Dig Dis Sci. 2009;54(11):2497-2502. doi:10.1007/s10620-008-0653-8

19. Peterson K, Carson S, Humphrey L, Helfand M. Patients with Positive Screening Fecal Occult Blood Tests: Evidence Brief on the Relationship between Time Delay to Colonoscopy and Colorectal Cancer Outcomes. Clin Gastroenterol Hepatol. 2016;14(10):14451451.e8.

20. Shah HA, Paszat LF, Saskin R, Stukel TA, Rabeneck L. Factors associated with incomplete colonoscopy: a population-based study. Gastroenterology. 2007;132(7):2297-2303. doi:10.1053/j.gastro. 2007.03 .032

21. Koido S, Ohkusa T, Nakae K, et al. Factors associated with incomplete colonoscopy at a Japanese academic hospital. World $J$ Gastroenterol. 2014;20(22):6961-6967. doi:10.3748/wjg.v20.i22. 6961

22. Palmer CK, Thomas MC, von Wagner C, Raine R. Reasons for nonuptake and subsequent participation in the NHS Bowel Cancer Screening Programme: a qualitative study. Br J Cancer. 2014;110 (7):1705-1711. doi:10.1038/bjc.2014.125

23. Jones RM, Devers KJ, Kuzel AJ, Woolf SH. Patient-reported barriers to colorectal cancer screening: a mixed-methods analysis. Am J Prev Med. 2010;38(5):508-516. doi:10.1016/j.amepre.2010.01.021 


\section{Supplementary materials}

Table SI Different stages of colorectal cancer at different response times among screening participants

\begin{tabular}{|c|c|c|c|}
\hline Time to examination & No. of cases (\%) & Adjusted odds ratio $(95 \% \mathrm{Cl})$ & Unadjusted odds ratio $(95 \% \mathrm{Cl})$ \\
\hline \multicolumn{4}{|l|}{ UICC stage I $(n=I .027)$} \\
\hline$\leq 30$ days & $109(1.0)$ & I (reference) & I (reference) \\
\hline $31-60$ days & $355(1.5)$ & $1.57(1.27-1.95)$ & $1.49(1.20-1.85)$ \\
\hline $6 I-90$ days & $189(1.8)$ & $2.16(1.70-2.74)$ & $1.89(1.49-2.40)$ \\
\hline$>90$ days & $374(3.2)$ & $3.58(2.89-4.45)$ & $3.28(2.65-4.10)$ \\
\hline \multicolumn{4}{|l|}{ UICC stage II $(n=82 I)$} \\
\hline$\leq 30$ days & $127(0.5)$ & I (reference) & I (reference) \\
\hline $31-60$ days & $279(1.2)$ & $1.05(0.85-1.30)$ & $1.00(0.81-1.24)$ \\
\hline $6 I-90$ days & $134(1.3)$ & $1.32(1.03-1.68)$ & $1.14(0.89-1.46)$ \\
\hline$>90$ days & $28 I(2.4)$ & $2.28(1.84-2.82)$ & $2.09(1.70-2.59)$ \\
\hline \multicolumn{4}{|l|}{ UICC stage III $(n=783)$} \\
\hline$\leq 30$ days & III (I.0) & I (reference) & I (reference) \\
\hline $31-60$ days & $288(1.2)$ & $1.23(0.98-1.53)$ & $1.19(0.95-1.48)$ \\
\hline $61-90$ days & $134(1.3)$ & $1.46(1.13-1.88)$ & $1.31(1.02-1.68)$ \\
\hline$>90$ days & $250(2.1)$ & $2.27(1.81-2.85)$ & $2.13(1.70-2.67)$ \\
\hline \multicolumn{4}{|l|}{ UICC stage IV $(n=259)$} \\
\hline$\leq 30$ days & $47(0.4)$ & I (reference) & I (reference) \\
\hline $31-60$ days & $92(0.4)$ & $0.94(0.66-1.34)$ & $0.89(0.63-1.27)$ \\
\hline $61-90$ days & $44(0.4)$ & $1.17(0.77-1.76)$ & I.0I (0.67-I.53) \\
\hline$>90$ days & $76(0.6)$ & $1.66(1.15-2.40)$ & $1.52(1.05-2.18)$ \\
\hline
\end{tabular}

Note: Adjusted for gender, year and age.

Abbreviation: UICC, Union for International Cancer Control.

Table S2 Sensitivity analysis on time and odds of finding cancer on conclusive examination

\begin{tabular}{|c|c|c|c|c|}
\hline \multirow[t]{2}{*}{ Time to FIT from invite } & \multicolumn{2}{|c|}{ Any colorectal cancer } & \multicolumn{2}{|c|}{ Advanced stage colorectal cancer } \\
\hline & No. of cases (\%) & Odds ratio $(95 \% \mathrm{Cl})$ & No. of cases (\%) & Odds ratio $(95 \% \mathrm{Cl})$ \\
\hline$\leq 30$ days & $2,4 \mid 2(6.3)$ & I (reference) & $698(1.8)$ & I (reference) \\
\hline $31-60$ days & $821(5.5)$ & $0.99(0.91-1.07)$ & $231(1.5)$ & $0.96(0.82-1.12)$ \\
\hline $6 I-90$ days & $315(5.8)$ & $1.06(0.94-1.20)$ & $103(1.9)$ & $1.19(0.97-1.47)$ \\
\hline$>90$ days & $184(5.2)$ & $1.05(0.90-1.23)$ & $51(1.4)$ & $0.99(0.74-1.32)$ \\
\hline \multicolumn{5}{|l|}{ Time to OC from pos. FIT-test } \\
\hline$\leq 30$ days & $\mathrm{I}, 868(4.2)$ & I (reference) & $635(1.4)$ & I (reference) \\
\hline $31-60$ days & $579(10.1)$ & $2.49(2.56-2.75)$ & $161(2.8)$ & $1.93(1.62-2.30)$ \\
\hline $6 \mathrm{I}-90$ days & $220(I I . I)$ & $2.68(2.31-3.10)$ & $57(2.9)$ & $1.92(1.46-2.53)$ \\
\hline$>90$ days & $972(20.1)$ & $5.32(4.89-5.79)$ & $189(3.9)$ & $2.59(2.19-3.06)$ \\
\hline
\end{tabular}

Note: Adjusted for gender, year, and age.

Abbreviations: FIT, Fecal Immunochemical Test; OC, Optical Colonoscopy. 


\section{Publish your work in this journal}

Clinical Epidemiology is an international, peer-reviewed, open access, online journal focusing on disease and drug epidemiology, identification of risk factors and screening procedures to develop optimal preventative initiatives and programs. Specific topics include: diagnosis, prognosis, treatment, screening, prevention, risk factor modification,

Submit your manuscript here: https://www.dovepress.com/clinical-epidemiology-journal systematic reviews, risk \& safety of medical interventions, epidemiology \& biostatistical methods, and evaluation of guidelines, translational medicine, health policies \& economic evaluations. The manuscript management system is completely online and includes a very quick and fair peer-review system, which is all easy to use. 